\title{
Carnets
}

Revue électronique d'études françaises de l'APEF

Deuxième série - $10 \mid 2017$

Pratiques de l'errance, vécus de la mémoire

\section{Petite note sur notre condition d'exilés}

\section{Cristina Robalo Cordeiro}

\section{(2) OpenEdition}

Journals

Édition électronique

URL : http://journals.openedition.org/carnets/2280

DOI : $10.4000 /$ carnets. 2280

ISSN : 1646-7698

Éditeur

APEF

\section{Référence électronique}

Cristina Robalo Cordeiro, «Petite note sur notre condition d'exilés 》, Carnets [En ligne], Deuxième série - 10 | 2017, mis en ligne le 30 avril 2017, consulté le 02 mai 2019. URL : http:// journals.openedition.org/carnets/2280 ; DOI : 10.4000/carnets.2280

Ce document a été généré automatiquement le 2 mai 2019.

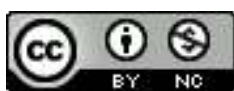

Carnets est mis à disposition selon les termes de la licence Creative Commons - Atribution - Pas d'utilisation commerciale 4.0 International. 


\title{
Petite note sur notre condition d'exilés
}

\author{
Cristina Robalo Cordeiro
}

1 Je confesse que, par un effet de l'âge ou de l'époque, il m'est devenu presque impossible aujourd'hui de séparer ma réflexion sur un thème comme l'exil d'un retour sur notre métier de professeur de littérature.

2 Placée, il y a trente ans, devant un tel «motif littéraire », je me serais aussitôt mise en devoir, dans un premier temps, de dresser un inventaire, de rassembler un corpus, bref de réduire l'exil à un « lieu » rhétorique, situé, sous le rapport des valorisations affectives, aux antipodes $\mathrm{du}$ « locus amoenus » mais susceptible du même type d'analyse.

Du reste, le travail d'inventaire et d'organisation m'aurait été largement facilité par un de ces petits ouvrages pédagogiques que les directeurs de collections ont multipliés à partir des années 1960 et où il était traité, par exemple, dans le volume 701 de La Révolte, dans le 705 du Suicide, dans le 709 de L'Exotisme et ainsi de suite... Toute l'expérience humaine, telle que la littérature en a fait sa matière, s'est ainsi bientôt trouvée cataloguée chez Bordas, Hatier ou Hachette, sous des rubriques brutalement claires. Que vous vous intéressiez à l'Argent ou au Mal, il vous suffisait, il vous suffit encore, de consulter la liste de ces publications à usage scolaire pour avoir le sentiment très rassurant de tenir en une dizaine de recueils les diverses expressions poétiques ou romanesques des principaux affects, biens ou maux, intéressant le cœur de l'Homme.

Que tout se réduise, selon les modes et courants de l'épistémologie littéraire, à des thèmes ou à des figures, à des réseaux sémantiques ou à des tropes, la tentation reste la même d'enfermer l'humain dans une combinatoire dont ne peut jamais sortir rien d'absolument nouveau ou, pour reprendre l'extraordinaire formule mallarméenne, de faire tenir «le mystère du monde... dans un Premier-Paris du Figaro » (Valéry, 1957 : 708). L'avantage de l'approche thématique sur la doctrine formaliste reste peut-être celui de préserver un certain degré d'initiative chez les écrivains futurs qui, sur le même thème, pourront se livrer à des variations presque infinies, tandis que, de son côté, la "poétique » veut contenir le jeu de la création dans un nombre très limité de figures. N'est-il pas ironique néanmoins de constater que même "la rêverie d'évasion » se voit sagement circonscrite 
dans le répertoire des motifs littéraires susceptibles de «satisfaire les lecteurs d'aujourd'hui $»^{1}$. Il va de soi que l'exil apparaît en bonne place dans ces listes de thèmes proposés par les éditeurs et que de zélés collègues ont pris soin d'illustrer et de présenter à travers un choix de « morceaux » dûment annotés et commentés.

Il m'aurait donc suffi jadis, pour m'acquitter, aussi modestement qu'honorablement, de mon devoir de professeur, de m'emparer de l'une de ces anthologies qui, d'Ovide à Hugo, des Tristia aux Contemplations, de Baudelaire à Camus, recouvrent tout le terrain de l'exil littéraire classique et nous procurent la troublante sensation d'avoir épuisé par avance toutes les modulations des chants possibles inspirés par le mal d'absence. Observons qu'il ne me serait toutefois pas loisible, par les règles du jeu de la laïcité scolaire, de recourir à la Bible et à sa riche veine exilique : c'est donc bien à regret que l'enseignant rémunéré par l'Etat devra délaisser les fleuves de Babylone du Psaume 136 ainsi que la très riche méditation théologique sur la signification de l'exil dans le dessein de Dieu pour s'en tenir à un florilège profane.

6 Délaissant les textes, toujours singuliers et d'autant plus irréductibles à l'analyse qu'ils sont plus forts, je m'en tiendrai pour l'heure aux idées générales, beaucoup plus faciles à traiter de manière expéditive. Je me demanderai ainsi dans un premier moment ce qu'il en est du concept d'exil dans un monde ramené aux proportions d'un « village global ».

7 Force nous est bien d'admettre en effet que la technologie de la communication, la rapidité des transports, le franchissement électronique des frontières menace le statut symbolique, sinon la réalité physique de l'exil. Avec la conversation par skype ou tout autre type de liaison par internet, l'éloignement a perdu beaucoup de son caractère douloureux. Si l'amour, d'après la jolie définition de Marcel Proust, est «l'espace et le temps rendus sensibles au cœur ", reconnaissons que l'amour au Xxi ${ }^{e}$ siècle ne peut plus régner que sur la moitié de son empire, la distance physique ayant été en partie abolie par la transmission immédiate du son et de l'image, en attendant mieux. Quant au temps, c'est sans doute un autre problème, que nous reprendrons un peu plus bas.

8 L'idée d'exil souffre d'une autre mutation, liée assurément à la transformation du monde par les applications concrètes du savoir scientifique : la notion de patrie a évolué à tel point que, au moins dans les couches socio-professionnelles supérieures, le cosmopolitisme n'est plus, comme au $18^{\mathrm{e}}$ siècle, un idéal mais un état de fait. Or l'exil est défini par le dictionnaire comme «la situation de quelqu'un expulsé de sa patrie »: si le sentiment de patrie disparait ou s'atténue, l'exil perd beaucoup de son sens.

9 J'ouvrirai une brève parenthèse. Sans doute parle-t-on aujourd'hui plus que jamais du sort dramatique des réfugiés comme des populations victimes de déportations en masse. Mais ici la misère atteint un tel degré que le terme d'exil apparaît faible et comme nimbé - ou entaché ? - de littérature. Il faut à ce propos préciser que le sentiment d'exil s'apparente à celui de solitude : un exil collectif (comme celui des juifs en Babylonie) correspond à une époque où l'individu n'avait pas encore conquis le statut exclusif que lui accordera la modernité. Et si l'exil, au sens actuel du mot, est bien la situation de quelqu'un, il est douteux que ce terme puisse s'appliquer en toute rigueur au déplacement de groupes entiers.

10 J'irai même jusqu'à relever l'écart entre la valeur héroïque ou sublime qu'a pu revêtir la notion d'exil dans la tradition et le soupçon qui pèse de nos jours sur le particularisme aliénant des petites communautés d'immigrés repliées sur elles-mêmes et leur culture. On 
se souviendra à cet égard du discours proféré par V. S. Naipaul recevant le prix Nobel de littérature en 2001 :

A Trinidad, où, nouveaux arrivants, nous formions une communauté désavantagée, cette idée d'exclusion était une sorte de protection, qui nous permettait, pour un moment seulement, de vivre à notre manière et selon nos propres règles, de vivre dans notre propre Inde en train de s'effacer. ${ }^{2}$

11 C'est en rompant avec cet égocentrisme insulaire de l'exil que Naipaul, devenu « voyageur » s'est ouvert l'accès à la « pensée élargie ». Voyageur, c'est-à-dire nomade, au sens où le définissait Jacques Attali en 1998 dans son Dictionnaire du XXI siècle :

L'archétype humain du siècle prochain. Ses valeurs, ses idées, ses désirs domineront

la société. Le marché fera tout pour le satisfaire, lui permettre d'emporter sa

maison avec lui et de rester connecté avec les principales oasis. (Attali, $1998: 232$ )

12 Autant dire que l'exil, dans cette vision futuriste, n'aura bientôt plus sa place sur la planète ou qu'il ne survivra que dans un imaginaire romantique. Car, il y a un pathos de l'exil, esthétiquement valorisé, qui nous interdit de tout mélanger: convenons par conséquent de considérer, dans le cadre qui est le nôtre, la seule littérature de l'exil avant d'évoquer l'exil de la littérature - ce qui est bien notre affaire à nous, professeurs de littérature.

13 Notons que si les prodiges techniques ont porté un coup sévère, mortel peut-être, à la symbolique de l'exil, la philosophie nietzschéenne avait déjà ébranlé les bases de l'édifice métaphysique avec lequel le sentiment d'exil entretenait une étroite dépendance. Nous nous situons avec l'auteur de Zarathoustra sur le plus large plan, à la base de nos valeurs et croyances: le sentiment universel d'exil, au sens extrapolitique du mot, ne peut être interprété que comme résultant du nihilisme accablant le vieil homme. C'est depuis Zénon, le « cruel Zénon », inventeur de la métaphysique, que la raison se sent exilée dans le temps et le mouvement du monde sublunaire. Le platonisme et le christianisme, ces deux figures majeures de l'idéalisme, ont fait de la condition humaine, et en premier lieu de notre condition corporelle, un exil originaire. Reconnaissons que la pensée de Nietzsche n'a fait qu'annoncer ce que la technologie a réalisé : la suppression de l'arrièremonde et, par entraînement, de ce «mal du retour » qui faisait écrire à Du Bellay :

Que songes-tu, mon âme emprisonnée?

Pourquoi te plaît l'obscur de notre jour,

Si pour voler en un plus clair séjour,

Tu as au dos l'aile bien empennée ? (apud Allem, 1965 : 335)

14 Un enseignant expliquant l'Idée à son public d'élèves munis de smartphones devra se montrer très ingénieux et très indulgent. Peut-être parviendra-t-il à arracher ses auditeurs au confort de l'immanence (pour ne pas employer le mot « matérialisme », dont on ne sait plus au vrai ce qu'il peut vouloir dire) en leur demandant d'esquisser une psychologie du Regret. C'est que si l'espace a été vaincu par les exploits technologiques, le temps résiste toujours, la mémoire persiste à accuser le manque, l'impatience atteste encore la réalité de la séparation entre deux êtres, entre l'être et son désir.

15 J'indiquerai au passage que le bergsonisme est venu en somme confirmer le refus de voir le temps comme la punition d'une faute originelle. Ainsi que l'écrit Jankélévitch, chez Bergson, avec Bergson, «le devenir n'est plus la vallée de larmes d'où l'homme, pèlerin perpétuel, ne songe qu'à s'enfuir; l'homme n'est plus un exilé ici-bas. » (Jankélévitch, 1959 : 268). Je me suis autrefois attachée à montrer comment un poète et conteur comme J. Supervielle faisait de l'éternité et de l'immobilité une espèce de châtiment où les vivants connaissent le seul véritable exil. 
Mais nous savons combien il est devenu difficile de faire aimer la littérature dans toute sa pauvreté de moyens (et la numérisation ne modifie pas son essence langagière). C'est pourquoi il m'apparaît de plus en plus nettement, à mesure que le développement électronique nous emporte loin de nos sources morales et esthétiques, que l'exil ne sera bientôt plus que celui du professeur de littérature lui-même, où qu'il soit (tandis qu'il épargne, dans une certaine mesure, ses collègues enseignants d'histoire ou de philosophie). Nous tentons de faire aimer la littérature et les grands textes du patrimoine littéraire comme des exilés s'efforcent, bien vainement, de communiquer leur nostalgie en exaltant les beautés de leur royaume perdu. L'enseignement de la littérature est notre Exil : exilés de l'intérieur et rois déchus nous ne vivons que de la protection de l'Etat, dont on peut craindre, dans un monde devenu le meilleur des mondes, qu'elle ait une fin prochaine. A tout le moins aurons-nous eu l'honneur d'être les derniers Exilés.

\section{BIBLIOGRAPHIE}

ALLEM, Maurice (1965). Anthologie poétique française. XVI $I^{\grave{m} e}$ siècle 1. Paris : Garnier-Flammarion.

ATTALI, Jacques (1998). Dictionnaire du XXIe siècle. Paris : Fayard.

FERRY, Luc (2009). Paroles de Philosophe. Paris : Dalloz.

JANKÉLÉVITCH, V. (1959). Henri Bergson. Paris : PUF.

VALÉRY, Paul (1957). Cuvres. Paris : Gallimard, Bibliothèque de la Pléiade, t. I.

\section{NOTES}

1. Dos de couverture de la collection Univers des lettres /Bordas, 1970.

2. V. S. Naipaul, «Comment je suis devenu écrivain, Deux mondes : discours de réception du prix Nobel», (novembre 2001), cité par Luc Ferry (FERRY, $2009: 196)$.

\section{RÉSUMÉS}

Avant la crise morale qui le touche aujourd'hui le professeur de littérature jouissait, grâce à un outillage pédagogique abondamment fourni par les éditeurs, d'une incontestable maitrise sur l'ensemble des grands "thèmes » traités par les écrivains. Mais l'exil, tout prestigieux qu'il apparaissait dans les catalogues scolaires, n'est plus ce qu'il était. Les transformations de notre environnement et la réduction, voire l'abolition, des distances, l'effacement de l'idée même de patrie, accusent de plus en plus le caractère littéraire et symbolique de l'exil. S’il reste quelques 
exilés authentiques dans le monde globalisé, ne faut-il pas aller les trouver là où l'on cherche encore à enseigner la littérature et parmi les professeurs que nous sommes?

Before the moral crisis attaining him/her in our present days, the teacher of literature, used to wield a real power over the whole variety of themes treated by generations of writers, such as Exile. But things have changed. Exile, as prestigious as it appeared in the catalogues of the publishers, is no longer what it was! The striking shortening of distances, the quick fading of the very idea of « homeland », denounces the literary and symbolic essence of Exile. If some genuine exiled people still exist in our globalized world, they are to be found in the class-rooms where literature is taught, among us, teachers.

\section{INDEX}

Mots-clés : enseignement de la littérature, thèmes, technologie, symbolique, idéalisme

Keywords : literature teaching, themes, technology, symbolic, idealism

\section{AUTEUR}

CRISTINA ROBALO CORDEIRO

Universidade de Coimbra

crobalo[at]@uc.pt 